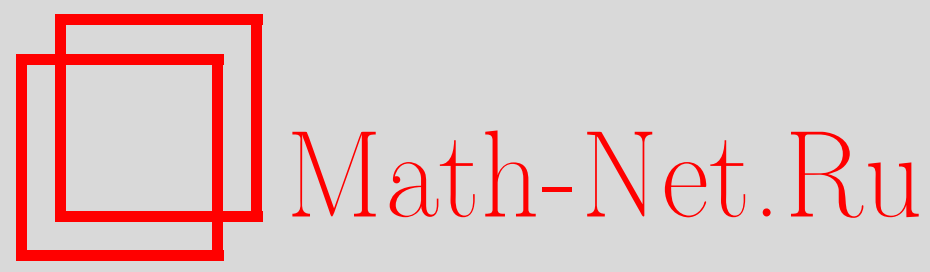

Б. Ю. Стернин, В. Е. Шаталов, Метод перевала и ресургентный анализ, Матем. заметки, 1997, том 61, выпуск 2, 278-296

DOI: https://doi.org/10.4213/mzm1501

Использование Общероссийского математического портала Math-Net.Ru подразумевает, что вы прочитали и согласны с пользовательским соглашением http://www . mathnet.ru/rus/agreement

Параметры загрузки:

IP : 54.157 .27 .8

26 апреля 2023 г., $17: 53: 47$ 


\title{
МЕТОД ПЕРЕВАЛА И РЕСУРГЕНТНЫЙ АНАЛИЗ
}

\author{
Б. Ю. Стернин, В.Е. Шаталов
}

Как известно, топологическая часть теории параметрического интеграла Лапласа разделяется на два этапа: первый состоит в разложении контура интегрирования в сумму контуров наискорейшего спуска при некотором значении параметра. На втором этапе осуществляется продолжение этого разложения (и, как следствие этого, продолжение асимптотического разложения рассматриваемого интеграла) на все оставшиеся значения параметра. В работе средствами ресургентного анализа исследуется второй этап этой задачи.

Библиограф̆ия: 13 названий.

Мы рассмотрим применение метода ресургентного анализа для исследования асимптотического поведения так назьваемых интегралов Лапласа вида

$$
I(x, k)=\int_{\gamma(x)} e^{k S(x, y)} a(x, y) d y .
$$

Здесь $k$ - большой параметр, $x=\left(x^{1}, \ldots, x^{n}\right) \in \mathbb{C}^{n}$ - комплексные параметры, которые принадлежат компактному множеству в пространстве $\mathbb{C}^{n}, y=\left(y^{1}, \ldots, y^{m}\right) \in \mathbb{C}^{m}$ и интегрирование проводится по некоторому $m$-мерному контуру $\gamma$ в комплексной плоскости $\mathbb{C}^{m}$, который может зависеть от параметров $x$ регулярньм образом (более точно, контур $\gamma(x)$ есть представитель некоторого класса гомологий $h(x)$, см. ниже).

Мы покажем, что теория интегралов такого типа есть частньй случай так называемого преобразования Бореля-Лапласа

$$
\mathscr{L}[F(s, x)]=\int_{\Gamma} e^{-k s} F(s, x) d s .
$$

Более точно, будет показано, что интегралы типа (1) (при некоторых дополнительных предположениях) определяют ресургентную функцию параметра $k$, зависящую от параметра $x \in \mathbb{C}^{n}$ (см. [1]-[4]).

Поясним вкратце понятие ресургентной функции (точные определения читатель может найти в цитированных вьше работах). Предположим, что функция $f(k, x)$ может быть представлена в виде (2), причем подынтегральная функция $F(s, x)$ является бесконечно-продолжимой функцией с простыми особенностями. Последнее

Работа выполнена при поддержке Российского фонда фундаментальных исследований, грант № 95-01-01306. 
означает, что $F(s, x)$ имеет при каждом фиксированном $x$ дискретное множество особенностей $s=S_{j}(x), j=1,2, \ldots$, на своей римановой поверхности и в окрестности каждой точки особенности представима в виде

$$
F(s, x)=\frac{a_{0}(x)}{s-S_{j}(x)}+\ln \left(s-S_{j}(x)\right) \sum_{l=0}^{\infty} \frac{\left(s-S_{j}(x)\right)^{l}}{l !} a_{l+1}^{(j)}(x),
$$

причем ряд, стоящий в правой части последнего соотношения, сходится в некоторой окрестности точки $s=S_{j}(x)$. Тогда асимптотика рассматриваемой функции $f(k, x)$ имеет вид

$$
f(k, x) \simeq e^{-k S_{j}(x)} \sum_{l=0}^{\infty} k^{-l} a_{l}^{(j)}(x) .
$$

Таким образом, если некоторая функция $f(k, x)$ представлена в виде суммы интегралов вида (2) по контурам $\Gamma_{j}$, охватьвающим некоторые особенности $s=S_{j}(x)$, то асимптотика этой функции имеет вид

$$
f(k, x) \simeq \sum_{j} e^{-k S_{j}(x)} \sum_{l=0}^{\infty} k^{-l} a_{l}^{(j)}(x)
$$

причем сумма берется по некоторому дискретному множеству точек $s=S_{j}(x)$ в комплексной плоскости $\mathbb{C}_{s}$ (отметим, что ряды, стоящие в правой части соотношения (3), являются расходящимися, и для корректной интерпретации асимптотического разложения их необходимо суммировать). В этом смысле функция $f(k, x)$ является функцией с дискретными асимптотиками. Методы ресургентного анализа, описанные в цитированных выше книгах, позволяют определять, какие особенности функции $F(s, x)$ участвуют в асимптотическом разложении (3) (активные и пассивные точки особенности), в каких точках $x$ активная особенность может стать пассивной и наоборот (явление Стокса), а также дают способ суммирования расходящихся рядов.

Более того, представление функции, определенной интегралом типа (1) в виде ресургентной функции, позволяет построить теорию асимптотических разложений таких интегралов с точностью до экспоненциально убьвающих членов. В этой связи мы упомянем работы Майкла Берри и Криса Хоулса (см., например, [5]), где соответствующая теория строится на основе введенного ими понятия гиперасимптотик. Отметим, однако, что эта теория также опирается на ресургентные представления функций, описанные в настоящей статье.

В данной статье авторы используют некоторую идею Мальгранжа [6] и Ф. Фама [7], которая состоит в том, что интеграл (1) представляется в виде преобразования Лапласа функции $F(s, x)$, полученной из амплитуды $a(x, y)$, интегрированием по исчезающему циклу поверхности $\{s=S(x, y)\}$. В работе [7] эта идея была использована для получения асимптотического разложения интеграла типа (1), взятого по специальному контуру $\gamma(x)$ (так называемому наперстку Лефшеца). Мы используем это представление для изучения явления Стокса в интегралах типа (1).

Содержание данной работы явилось предметом цикла лекций, которые авторы читали в рамках программы "Ecole Doctorale" в июне 1996 г. в Университете Ниццы (Франция). Авторы признательны Фредерику Фаму и Крису Хоулсу за ряд полезных обсуждений, имевших место в период проведения этой школы. 
Авторы также выражают свою благодарность Владимиру Назайкинскому за весьма полезные дискуссии по теме данной статьи. Нам также хотелось бы поблагодарить Александра Чеботарева за ряд полезных замечаний,несомненно способствовавших лучшему пониманию статьи. Английская версия настоящей работы опубликованав качестве препринта [8].

1. Постановка задачи. Предположим для простоты, что контур $\gamma$, участвующий в определении интеграла (1) выбран таким образом, что для достаточно большого $|y|$ справедливо неравенство $\operatorname{Re} S>-c_{1}|y|$ с некоторой положительной константой $c_{1}$ и что функция $a(x, y)$ имеет экспоненциальньй тип по $y$, т.е. вьполнено неравенство

$$
|a(x, y)| \leqslant C e^{c_{2}|y|}
$$

с некоторыми постоянными $C>0$ и $c_{2}$. При этих условиях интеграл (1) сходится для достаточно больших значений параметра $k$. Мы предположим также, что все стационарные точки функции $S(x, y)$ невырождены.

Вычислим вначале экспоненциальньй тип интеграла (1). Имеем, очевидно,

$$
|I(x, k)| \leqslant C_{1} e^{M(x) k},
$$

где

$$
M(x)=\sup _{y \in \gamma(x)} \operatorname{Re} S(x, y)
$$

Однако, последняя оценка является весьма грубой, поскольку можно заменить контур интегрирования $\gamma(x)$ на контур из того же класса гомологий и при этом интеграл $I(x, k)$ не изменится, а правая часть равенства (1), вообще говоря, станет иной.

В действительности, интегрирование в (1) происходит по классу гомологий $h(x)$ (см. [9]), которьй уходит в бесконечность вдоль направления убьвания функции $\operatorname{Re} S(x, y)$, а не по какому-то конкретному контуру $\gamma(x) \in h(x)$. Таким образом, оценка (4) может быть уточнена следующим образом:

$$
M(x)=\inf _{\gamma^{\prime}(x) \in h(x)}\left(\sup _{y \in \gamma^{\prime}(x)} \operatorname{Re} S(x, y)\right)
$$

где точная нижняя грань берется по всем $m$-мерньг контурам, гомологичньм контуpy $\gamma(x)$.

Эти рассуждения показьвают, в частности, что для исследования асимптотических свойств интеграла $I(x, k)$ удобно выбрать представителя $\gamma(x)$ в классе гомологий $h(x)$ таким образом, чтобы функция $\operatorname{Re} S(x, y)$ была на этом контурекак можно меньше. Для того, чтобы сформулировать точные требования на выбор контура $\gamma(x)$, введем понятие контура наискорейшего спуска.

Обозначим через $X=\operatorname{grad} \operatorname{Re} S(x, y)$ (вещественньй) градиент функции $\operatorname{Re} S(x, y)$ в комплексном пространстве $\mathbb{C}^{m}$. Тогда контур наискорейшего спуска есть, грубо говоря, контур, который содержит как минимум одну точку перевала (седловую точку) функции $S(x, y)$ и который касается векторного поля $X$ так, что функция $\operatorname{Re} S(x, y)$ убьвает вдоль траектории этого векторного поля. Для того, чтобы уточнить это определение, мы должны принять также во внимание структуру контура вблизи седловых точек, поскольку эти точки являются особыми точками поля $X$. 
Поскольку предполагается, что все стационарные точки функции $S(x, y)$ невырождены (в силу условий Коши-Римана эти точки совпадают, очевидно, с седловьми точками функции $\operatorname{Re} S(x, y))$, можно легко проверить, что каждая седловая точка $y_{0}$ функции $\operatorname{Re} S(x, y)$ является гиперболической точкой и ее отталкивающее подпространство $L_{-}$имеет размерность $m$. Теперь контур наискорейшего спуска определяется как $m$-мерный контур, инвариантньй относительно векторного поля $X$, содержащий, по крайней мере, одну седловую точку $y_{0}$ и касательньй в этой точке $L_{-}$.

В общем положении каждьй контур наискорейшего спуска содержит одну седловую точку функции $\operatorname{Re} S(x, y)$. Однако, если параметр $x$ меняется, то для некоторых значений $x$ контур наискорейшего спуска может изменить свою топологическую структуру, и, как мы увидим ниже, эта ситуация в точности соответствует явлению Стокса для интегралов типа (1).

Наиболее прозрачньми предыдущие рассмотрения становятся в одномерном случае (т.е. для $m=1$ ). В этом случае мы можем представить себе, что контур $\gamma$ является веревкой, положенной на поверхность графика функции $\operatorname{Re} S(x, y)$. Продеформируем эту веревку (не изменяя значения интеграла (1)) таким образом, чтобы она располагалась так низко, как только возможно на рассмотренной поверхности. В результате этого веревка повиснет на седловой точке функции $\operatorname{Re} S(x, y)$, выходя из этой точки в направлении градиента $X$ функции $\operatorname{Re} S(x, y)$. Дальнейшее положение веревки будет однозначно определенным, если только интегральная кривая поля $X$, вдоль которой веревка располагается, не встретит другую седловую точку функции $\operatorname{Re} S(x, y)$. В этом последнем случае оставшаяся часть контура наискорейшего спуска может иметь два различных направления, соответствующих двум отталкивающим направлениям поля $X$, и конструкция контура становится неоднозначной. Значения параметра $x$, для которых подобная ситуация имеет место, являются в точности точками топологической перестройки контура наискорейшего спуска (линией Стокса соответствующего интеграла).

Мы заметим, что в одномерном случае мнимая часть функции $S(x, y)$ постоянна вдоль любого контура наискорейшего спуска. Обратно, если мнимая часть функции $S$ постоянна вдоль некоторого контура $\gamma$, то этот контур касателен введенному вьше векторному полю $X$.

Проведенные вьше рассуждения показьвают, что метод перевала построения асимптотических разложений типа (1) состоит из двух отдельных частей:

1) топологической части этой теории, цель которой исследовать контуры интегрирования и редуцировать рассматриваемьй интеграл к сумме интегралов вдоль контуров наискорейшего спуска;

2) аналитической части, цель которой исследовать интеграл вдоль таких контуров и дать их асимптотическое разложение.

Вторая часть этой теории детально разработана, и читатель может найти ее в учебниках по методу перевала (см., например, [10]). Что касается первой (топологической) части этой теории, то в случае, когда интеграл зависит от некоторых дополнительных параметров $x$ (а это именно та ситуация, которую мы рассматриваем), эта часть исследования может быть разделена, в свою очередь, на два этапа. Первьй этап состоит в разложении контура интегрирования в сумму контуров наискорейшего спуска для некоторого фиксированного значения параметра, а на втором этапе осуществляется продолжение этого разложения (и, как следствие, продолжение асимптотического разложения рассматриваемого интеграла) на все оставшиеся значения параметра $x$. 
Ниже мы сконцентрируем наше внимание на втором этапе топологической части теории. Именно, мы предположим, что разложение контура интегрирования определено для некоторого значения параметра $x$, и исследуем такое разложение для всех других значений параметров $x$. Отметим, что в этом исследовании оказьвается весьма полезным метод ресургентного анализа.

Поскольку теория одномерных интегралов $(m=1)$ значительно проще многомерного случая, мы рассмотрим вначале этот простой случай, отложив исследование общей (многомерной) ситуации до п. 3.

2. Одномерный случай. Рассмотрим интеграл (1) для $y \in \mathbb{C}$, т.е. $m=1$. В этом случае интеграл берется по одномерному контуру $\gamma$. Будем считать выполненньми следуюшие предположения (эти ограничения не являются необходимьми, и в данном контексте мы предполагаем их выполненньми лишь для упрощения изложения).

1. Функция $S(x, y)$ является полиномом по $y$ с голоморфньми коэффициентами в области $D \subset \mathbb{C}^{n}$.

2. Для достаточно больших значений $|y|$ на контуре $\gamma$ выполнена оценка

$$
\operatorname{Re} S(x, y) \leqslant-c|y|
$$

с некоторой положительной константой $c$.

3. Функция $a(x, y)$ является целой положительной функцией экспоненциального типа порядка 1 по $y$ для $x \in D \subset \mathbb{C}^{n}$, т.е. имеет место неравенство

$$
|a(x, y)| \leqslant C e^{c|y|}
$$

с некоторыми постоянными $c$ и $C>0$.

Ясно, что если условия 1-3 выполнены, то интеграл (1) сходится для достаточно больших значений $k$ при любых $x \in D$ и определяет функцию $I(x, k)$ экспоненциального роста по $k$.

Для того чтобы привести интеграл (1) к виду преобразования Бореля-Лапласа (2) некоторой (гипер)функции [4], мы произведем замену переменных

$$
y=y(s, x),
$$

где функция $y(s, x)$ есть решение уравнения $s=-S(x, y)$. Поскольку в силу предположения 2 функция $S(x, y)$ не является тождественной константой по $y$ для каждого фиксированного значения $x \in D$, мы имеем $S^{\prime}(x, y) \not \equiv 0$, и поэтому функция $y(s, x)$ определяет бесконечно продолжимую аналитическую функцию конечного порядка, имеющую ветвление в каждой своей особой точке. (Определение бесконечно продолжимой аналитической функции см., например, в [2], [4]; часто слово “аналитическая" мы будем опускать; грубо говоря, бесконечно-продолжимая функция - это функция с дискретным множеством особенностей на ее римановой поверхности.) Кроме того, эта функция ограничена в окрестности точки ветвления. Замена (5) приводит интеграл (1) к функции $I(x, k)$ вида

$$
I(x, k)=\int_{\gamma^{*}} e^{-k s} b(s, x) d s
$$

по некоторому контуру $\gamma^{*}$ в плоскости $\mathbb{C}_{s}$, где

$$
b(s, x)=a(x, y(x, s)) \frac{\partial y(x, s)}{\partial s} .
$$


Следующие свойства представления (6) очевидны.

a) Функция $b(s, x)$, определенная соотношением (7), является бесконечно продолжимой функцией с интегрируемой особенностью в каждой своей точке ветвления.

b) Пересечение контура $\gamma^{*}$ с полуплоскостью $\operatorname{Re} s<A$ компактно при любом значении константы $A$. Другими словами, реальная часть $s$ стремится к $+\infty$ вдоль контуpa $\gamma^{*}$.

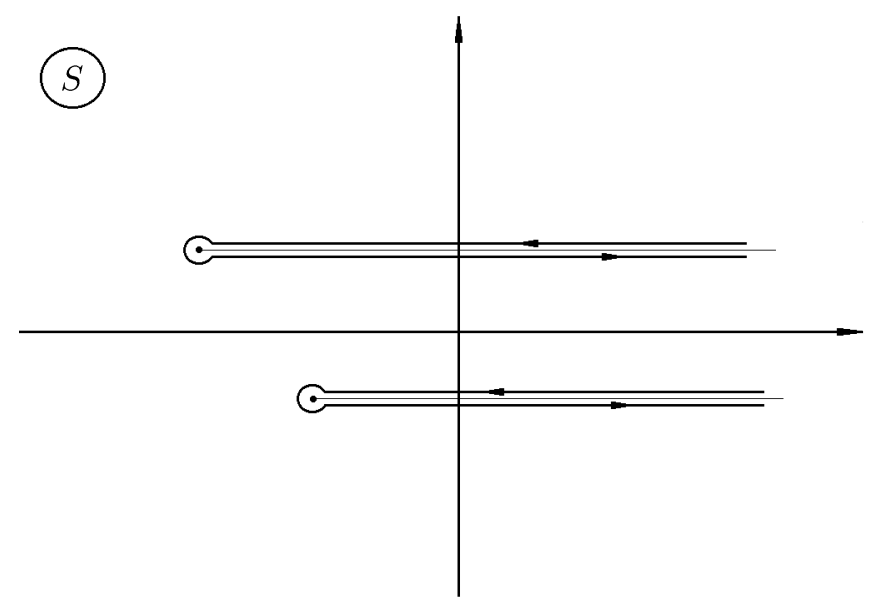

Рис. 1

Теперь мы можем деформировать контур $\gamma^{*}$, двигая его направо в комплексной плоскости $\mathbb{C}_{s}$. В результате такой деформации получим, что с точностью до быстроубываюших членов интеграл (6) имеет вид ресургентной функции, т.е.

$$
I(x, k)=\sum_{j} \int_{\Gamma_{j}} e^{-s} b(s, x, k) d s,
$$

где контур $\Gamma_{j}$ есть стандартньй контур, окружающий некоторую точку особенности $b(s, x)$ в направлении против часовой стрелки и уходящий в бесконечность вдоль направления положительной вещественной оси (см. рис. 1). Сумма в правой части последнего выражения берется по всем особым точкам функции $b(s, x)$, "видимым" из точек контуpa $\gamma^{*}$ вдоль лучей, направленных вдоль положительной вещественной оси.

Таким образом, доказано следующее утверждение.

Теорема 1. При сформулированных выше условиях 1-3 функиия $I(x, k)$, определенная интегралом (1), является ресургентной функиией, так что соответствующая гиперфункиия $b(s, x)$ регулярна и имеет алгебраическое ветвление в каждой своей особой точке.

Эта теорема позволяет применить методы ресургентного анализа к исследованию интегралов типа Лапласа.

Имеется еще одно свойство ресургентных функций, соответствующих интегралам типа (1), которое используется для исследования этих интегралов. Именно, из определения $I(x, k)$ и свойств $1-3$ следует, что функция $I(x, k)$ является однозначной функцией $x$ 
в области $D$. Поэтому соответствующая ресургентная функция должна удовлетворять ресургентным уравнениям в окрестности каждой своей фокальной точки (см., например, [4]).

Теорема 1 вместе с упомянутым выше замечанием позволяет достичь успеха при исследовании интегралов типа Лапласа в случае, когда эти интегралы зависят от некоторых дополнительных параметров $x$. Именно, если разложение контура интегрирования $\gamma$ в сумму контуров наискорейшего спуска известно для одного значения параметра, peсургентньй анализ позволяет строить такое разложение для всех оставшихся значений параметров. Опишем эту процедуру.

Вначале зафиксируем некоторое значение параметра $x$ и рассмотрим канонический контур $\Gamma$, соответствующий особой точке $s^{*}$ функции $b(s, x)$, заданной выражением (7). Этот контур состоит из двух лучей, выходящих из точки $s^{*}$ в направлении положительной вещественной оси, которая проходит в различньх направлениях и лежит на различных листах римановой поверхности функции $b(s, x)$ (такой контур может быть использован в силу того, что эта функция интегрируема в особой точке $s^{*}$ ). Поскольку мнимая часть функции $S$ постоянна вдоль этого контура, ясно, что при замене переменных $s=-S(x, y)$ (см. (5)) контур перейдет в контур наискорейшего спуска, проходящий через седловую точку, соответствующую точке $s^{*}$. Таким образом, мы получаем разложение (произвольного) контура $\gamma^{*}$ в интеграле (1) в сумму контуров наискорейшего спуска.

Далее, хорошо известно (см. [10]), что для некоторых значений параметров $x$ (на соответствующей поверхности Стокса) имеет место бифуркация структуры контура наискорейшего спуска. Более точно, контур наискорейшего спуска может перейти в сумму таких контуров, при которых точка $x$ пересекает соответствующую линию Стокса. Эта ситуация имеет место в случае, когда контур наискорейшего спуска, проходяший через некоторую седловую точку, приходит к некоторой другой седловой точке (такой феномен будет иметь место в случае, когда точка $x$ принадлежит поверхности Стокса). И затем имеется в точности две (для невырожденной стационарной точки) возможности продолжения контура наискорейшего спуска: его можно продолжить в одну из двух долин графика функции $\operatorname{Re} S(x, y)$. Поэтому контур наискорейшего спуска (а вместе с ним и асимптотическое разложение) меняется скачком, когда параметр $x$ пересекает поверхность Стокса.

В силу взаимно однозначного соответствия между контурами наискорейшего спуска в $y$-плоскости и каноническими контурами в $s$-плоскости описанная бифуркация контура наискорейшего спуска отвечает бифуркации соответствующего канонического контура в $s$-плоскости. Эта бифуркация в теории ресургентных функций есть в точности бифуркация, описьваемая при помощи связьвающего гомоморфизма (см., например, [4]). Последнее обстоятельство позволяет применить технику связьвающего гомоморфизма и ресургентных уравнений к исследованию бифуркаций контуров наискорейшего спуска в теории интегралов Лапласа. Пример такого исследования будет рассмотрен в последнем пункте.

В заключение этого пункта мы заметим, что класс ресургентных функций, полученных из интегралов типа Лапласа, может быть описан как класс таких ресургентных функций $f(x)$, чьи преобразования Бореля $F(s, x)$ могут быть униформизованы с помощью некоторой голоморфной подстановки $s=S(x, y)$. Мы напомним, что функция $F(s, x)$ назьвается униформизуемой подстановкой $s=S(x, y)$, если функция 
$F(S(x, y), x)$ является регулярной голоморфной функцией переменных $(x, y)$.

3. Многомерный случай. Теория бифуркаций контуров наискорейшего спуска может быть разработана в многомерном случае аналогично построенной выше теории для одномерных интегралов Лапласа, хотя в многомерном случае соответствие между каноническими контурами и контурами наискорейшего спуска несколько более сложно. Для того чтобы установить это соответствие, напомним некоторые факты из теории многомерных интегралов Лапласа (см., например, [10]).

Рассмотрим интеграл типа (1). Как и вьше, мы предположим (для простоты), что выполнены следующие условия.

1. Функция $S(x, y)$ является полиномом по переменным $y \in \mathbb{C}^{m}$ и $a(x, y)$ есть целая функция для каждого фиксированного $x \in D \subset \mathbb{C}^{n}$, где $D$ - некоторая область в комплексной области $\mathbb{C}^{n}$.

2. При достаточно большом значении $|y|$ на $m$-мерном контуре $\gamma$ имеет место неравенство

$$
\operatorname{Re} S(x, y) \leqslant-c|y|
$$

с некоторой положительной константой $c$.

3. Функция $a(x, y)$ имеет экспоненциальньй тип и порядок 1 . Это означает, что имеет место неравенство

$$
|a(x, y)| \leqslant C e^{c|y|}
$$

с некоторыми константами $c$ и $C>0$.

Пусть $x$ - такое значение параметра, что на каждом контуре наискорейшего спуска лежит в точности одна седловая точка функции $\operatorname{Re} S(x, y)$ (это означает, что точка $x$ не лежит на поверхности Стокса соответствующего интеграла), и все седловые точки функции $\operatorname{Re} S(x, y)$ невырождены.

Исследуем более детально структуру контура наискорейшего спуска вблизи соответствующей седловой точки.

Мы заметим, что в силу условий Коши-Римана любая седловая точка функции $\operatorname{Re} S(x, y)$ является стационарной точкой функции $S(x, y)$. Поскольку по предположению все такие точки невырождены, в силу леммы Морса существует такая голоморфная замена переменных $y=y(z)$, что справедливо соотношение

$$
S(x, y(z))=S_{0}+\sum_{j=0}^{m}\left(z^{j}\right)^{2}
$$

где $S_{0}$ - значение функции $S(x, y)$ в седловой точке, которая соответствует началу координат в $z$-плоскости. Тогда контур наискорейшего спуска вблизи рассматриваемой седловой точки может быть записан в $z$-координатах следующим образом:

$$
z^{j}=u^{j} \in \mathbb{R}, \quad j=1, \ldots, m .
$$

Заметим, что в силу условия 2 функция $\operatorname{Re} S(x, y)$ ограничена сверху на контуре интегрирования $\gamma$. Обозначим через $A$ верхнюю границу этого контура $\gamma$ :

$$
A \stackrel{\text { def }}{=} \sup _{y \in \gamma} \operatorname{Re} S(x, y) .
$$


Часть $\gamma_{A^{\prime}}$ контура $\gamma$, лежащая в области

$$
\Omega_{A^{\prime}}=\left\{y: \operatorname{Re} S(x, y) \geqslant A^{\prime}\right\}
$$

для любого $A^{\prime}<A$ определяет элемент относительно компактных гомологий

$$
\gamma_{A^{\prime}} \in H_{m, c}\left(\Omega_{A^{\prime}}, \operatorname{Re} S(x, y)=A^{\prime}\right)
$$

Как доказано в [10], элемент $\gamma_{A^{\prime}}$ может быть разложен в сумму

$$
\gamma_{A^{\prime}} \simeq \sum_{j} \bar{\gamma}_{j}
$$

где каждый контур $\bar{\gamma}_{j}$ соответствует некоторой седловой точке $y_{j}$ функции $\operatorname{Re} S(x, y)$ и совпадает с контуром наискорейшего спуска (8) в окрестности этой точки. Поэтому с точностью до функции экспоненциального типа $A^{\prime}$ мы имеем

$$
I(x, k)=\sum_{j} I_{j}(x, k)
$$

где

$$
I_{j}(x, k)=\int_{\bar{\gamma}_{j}} e^{k S(x, y)} a(x, y) d y
$$

ЗАмечание 1. Разложение (9) может быть получено также из результатов $\Phi . \Phi а-$ мa [7].

Теперь мы должны изучить структуру интеграла $I_{j}(x, k)$ вблизи соответствующей седловой точки. Рассмотрим часть этого интеграла, распространенного по контуру $\bar{\gamma}_{j}^{r}=\bar{\gamma}_{j} \cap\{|z| \leqslant r\}$, где $r$ - достаточно малое число. Тогда можно предположить, что контур $\bar{\gamma}_{j}^{r}$ описьвается в координатах $z$ уравнениями (8). Мы имеем

$$
\begin{aligned}
\int_{\bar{\gamma}_{j}^{r}} e^{k S(x, y)} a(x, y) d y & =\int_{|u| \leqslant r} e^{k S(x, y(u))} a(x, y(u)) \frac{D y(u)}{D u} d u \\
& =\int_{0}^{r} d \rho^{2} \int_{|u|=\rho} e^{k S(x, y(u))} a(x, y(u)) \frac{D y(u)}{D u} \omega
\end{aligned}
$$

где $\rho=|u|$ и $\omega$ - форма, определенная соотношением $d u=d \rho^{2} \wedge \omega$. Поскольку на контуре $\bar{\gamma}_{j}^{r}$ мы имеем $S=S_{0}^{j}+\rho^{2}$, где $S_{0}^{j}$ - значение функции $S(x, y)$ в седловой точке $y_{j}$, внутренний интеграл в правой части последней формулы может быть рассмотрен как интеграл по исчезающему циклу $h_{j}(s, x)$ на многообразии

$$
\Sigma_{s, x}=\{y: S(x, y)=-s\}
$$

для $s=S_{0}^{j}+\rho^{2}$. Поэтому интеграл в правой части формулы (10) может быть представлен в виде

$$
\int_{\bar{\gamma}_{j}^{r}} e^{k S(x, y)} a(x, y) d y=\int_{\gamma_{j}^{r}} e^{-k s}\left\{\int_{h_{j}(s, x)} a(x, y) \omega\right\} d s
$$


где $\gamma_{j}^{r}$ - отрезок длины $r$ луча, выходящего из точки $s=-S_{0}^{j}$ вдоль направления положительной вешественной оси, а форма $\omega$ определена соотношением $d y=d s \wedge \omega$.

Мы заметим, что точка $s=-S_{0}^{j}$ есть одна из особых точек функции

$$
\mathscr{F}_{S}[a](s, x) \stackrel{\text { def }}{=} \int_{h_{j}(s, x)} a(x, y) \omega,
$$

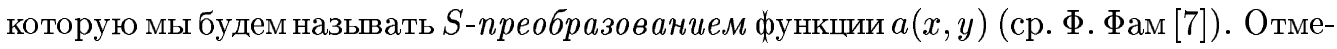
тим, что значение правой части последней формулы для различных значений $j$ представляет собой ветви одной и той же (ветвящейся) функции $\mathscr{F}_{S}[a](s, x)$. Мы заметим также, что при условиях 1-3 (см. выше) эта функция является бесконечно продолжимой.

Рассмотрим теперь интегралы

$$
I_{j}^{\prime}(x, k)=\int_{\gamma_{j}} e^{-k s \mathscr{F}_{S}}[a](s, x) d s
$$

для седловой точки $y_{j}$, входящей в разложение $(9)$. Здесь $\gamma_{j}-$ лучи, выходящие из критических значений $S_{0}^{j}$, соответствующих седловой точке и направленные вдоль положительной вещественной оси.

Заметим, что сходимость этих интегралов не необходима, поскольку их можно понимать в следующем смысле. Для любого вещественного числа $A$ выражения $I_{j}^{\prime}(x, k)$ являются интегралами одного и того же вида, взятьми по контурам, которые обрезаны при $\operatorname{Re} s=A$. Тогда в силу леммы Бореля (см., например, [11]) существует функция $I_{j}^{\prime}(x, k)$, которая совпадает с функцией с точностью до $O\left(e^{-A k}\right)$. Заметим также, что интеграл (11) может быть записан как интеграл типа (1) по так называемому наперстку Лефиеца (см. Ф. Фам [7] и С. Лефшец [12]). Эта функция и есть по определению значение рассматриваемого интеграла.

Проведенные вьше рассуж дения показьвают, что каждьй интеграл (11) может быть записан как интеграл вида (1), взятьй по контуру, которьй совпадает в окрестности соответствующей седловой точки с контуром наискорейшего спуска. Из разложения (9) мы видим, что с точностью до функции экспоненциального типа $A^{\prime}$ справедливо равенство

$$
I(x, k)=\sum_{j} I_{j}^{\prime}(x, k) .
$$

Это есть в точности ресургентное представление функции $I(x, k)$, поскольку интеграл (11) есть преобразование Лапласа бесконечно-продолжимой микрофункции, представленной своей вариацией $\mathscr{F}_{S}[a](s, x)$.

Суммируя полученные выше результаты, мы приходим к тому, что справедлива следующая теорема.

ТЕОРема 2. При условиях 1-3 интеграл (1) является ресургентной функиией. Соответствующая гиперфункиия определяется $S$-преобразованием амплитудной функиии $\mathscr{F}_{S}[a](s, x) a(x, y)$ интеграла $(1)$. Эта гиперфункиия регулярна и определяется соотв етствующ,ей вариачией.

Теперь исследование топологической части теории многомерных интегралов Лапласа вполне аналогично одномерному случаю, и мы оставляем его читателю. 


\section{4. Примеры.}

4.1. Интегралы типа Эйри. Здесь мы рассмотрим интегралы типа Эйри, т.е. интегралы Лапласа вида

$$
u(x, k)=\int_{\gamma} e^{k S(x, \xi)} a(x, \xi) d \xi
$$

(с контуром $\gamma$, удовлетворяющим указанньп вьше требованиям) в предположении, что фаза $S(x, \xi)$ имеет две седловые точки по переменной $\xi$, которые равны

$$
s=s(x)=\frac{2}{3} x^{3 / 2}
$$

Примером интегралов типа Эйри может служить функция Эйри

$$
A i(x, k)=k^{1 / 2} \int_{\gamma} e^{k\left(\xi x-\xi^{3} / 3\right)} d \xi
$$

где контур $\gamma$ может быть выбран так, как показано на рис. 2.

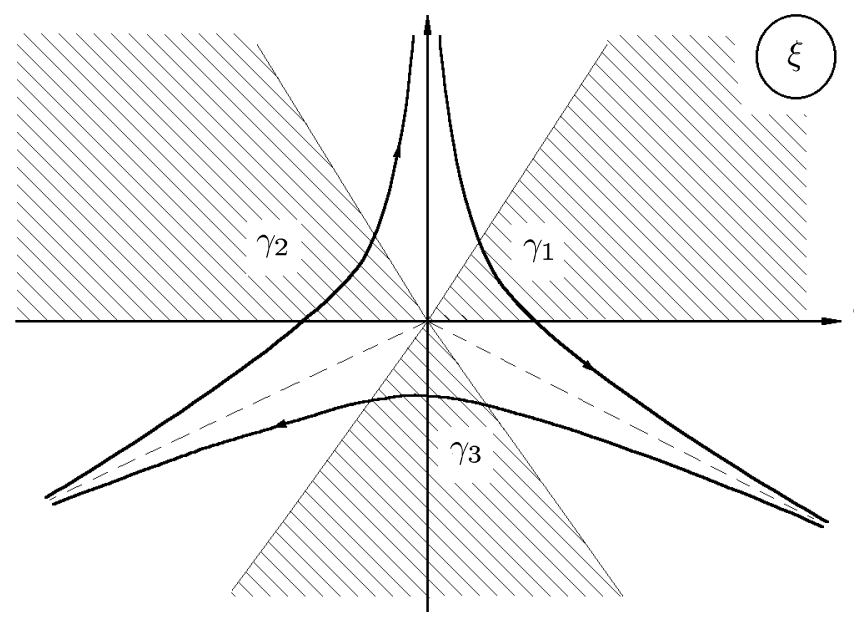

Рис. 2

Хорошо известно, что функция Эйри является решением следующего дифференциального уравнения:

$$
k^{-2} \frac{d^{2} u}{d x^{2}}-x u=0 .
$$

Делая замену переменных $\xi=\xi(s, x)$ так, что

$$
S(x, \xi)=-s
$$

мы приведем интеграл (12) к виду

$$
u(x, k)=\int_{\gamma^{\prime}} e^{-k s} F(s, x) d s,
$$


где

$$
F(s, x)=a(x, \xi(s, x)) \frac{d \xi(s, x)}{d s} .
$$

Заметим, что в силу сформулированных вьше предположений на критические точки функции $S(x, \xi)$ решение $\xi=\xi(s, x, k)$ уравнения (16) относительно $\xi$ является ветвящейся функцией переменной $s$ с особенностями, определенными формулами (13); два значения функции $s(x)$ мы обозначим через $s_{j}(x), j=1,2$. Очевидно, что для функции (12) фокальной точкой в $x$-плоскости является начало координат $x=0$ (мы напомним, что фокальные точки ресургентной функции - это точки ветвления функции $s(x)$, описьвающие особенность подьнтегрального выражения в представлении типа (17)). Поэтому единственное ресургентное уравнение, выражающее функцию (12), может быть записано на единичной окружности

$$
l=\left\{x=e^{i \varphi}\right\}, \quad \varphi \in[0,2 \pi] .
$$

Опишем соответствующую диаграмму освешения (см. [4]).

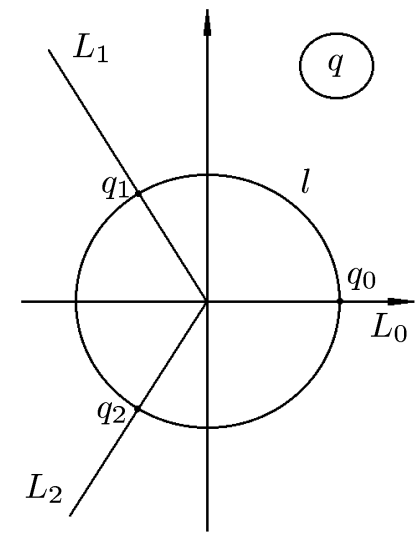

Рис. 3a)

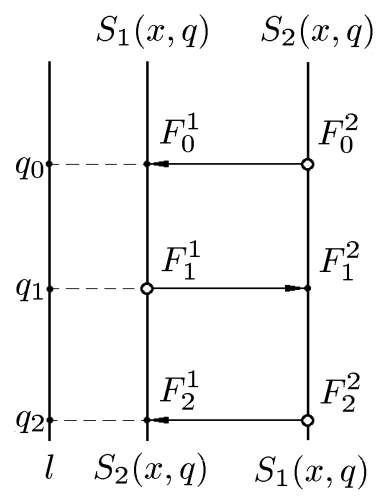

Рис. $3 \mathrm{~b})$

Эта диаграмма (см., например, рис. $3 \mathrm{~b}))$ содержит строки, соответствующие точкам $x_{1}, x_{2}, x_{3}$ пересечения рассматриваемого пути с поверхностями Стокса исследуемой функции. Для каждой такой точки одна из точек особенностей подынтегральной функции (17) лежит на луче, выходящем из другой точки особенности вдоль направления положительной вещественной оси; мы будем говорить, что последняя точка освещает первоначальную. Это показано на диаграмме с помощью стрелки, идущей из освещающей точки в освещаемую. Обозначим через $F_{i}^{j}$ микрофункцию, определяемую функцией $F\left(s, x_{i}\right)$ в точке $s=s_{j}\left(x_{i}\right)$.

Очевидно, одна из двух точек (13) освещает другую в том и только в том случае, когда $\varphi=0, \varphi=2 \pi / 3$ или $\varphi=4 \pi / 3$ (мы проводим наши рассмотрения для вешественных положительных значений $k$ ). Соответствующие линии Стокса изображены на рис. $3 \mathrm{a})$. Можно легко проверить, что диаграмма освещения имеет вид, указанный на рис. $3 \mathrm{~b}$ ). Следуя вдоль петли $l$, точки $s_{1}(x)$ и $s_{2}(x)$ меняют свое местоположение три раза. 
Нетрудно видеть, что соответствующая система ресургентных уравнений для диаграмм освещения этого типа (записанная для микрофункций $F_{0}^{1}, F_{1}^{2}$ и $F_{2}^{1}$, соответствующих освещенным точкам этой диаграммы) есть (впоследствии мы будем предполагать для краткости, что все сторонние производные, за исключением тех, которые включены в ресургентную систему явным образом, тождественно равны нулю)

$$
\left\{\begin{array}{l}
F_{0}^{1}=\mathscr{A}^{2} F_{1}^{2}-\Delta\left(\mathscr{A} F_{2}^{1}\right), \\
F_{1}^{2}=\mathscr{A}^{2} F_{2}^{1}-\Delta\left(\mathscr{A} F_{0}^{1}\right), \\
F_{2}^{1}=\mathscr{A}^{2} F_{0}^{1}-\Delta\left(\mathscr{A} F_{1}^{2}\right),
\end{array}\right.
$$

где $\Delta$ есть сторонняя производная (см. [1], [4]) и $\mathscr{A}$ есть оператор аналитического продолжения соответствующей микрофункции вдоль части петли $l$, расположенной между двумя последовательными точками пересечения этой петли с поверхностями Стокса.

Мы подчеркнем, что для любой данной гиперфункции $F(s, x)$, имеющей точки (13) в качестве точек ветвления, можно выбрать множество микрофункций $F_{0}^{1}, F_{1}^{2}$ и $F_{2}^{1}$, определенных особыми точками функции $F(s, x)$, таким образом, чтобы соответствующая ресургентная функция $u(x, k)$ была однозначна в окрестности начала и, следовательно, уравнения (18) были бы вьполнены. Для того чтобы это сделать, достаточно определить функцию $u(x, k)$ как интеграл вида (17) с контуром интегрирования $\Gamma$, окружающим обе точки ветвления так, как это показано на рис. 4. Тогда разложение полученной функции даст нам требуемую микрофункцию, которая удовлетворяет системе (18). Однако, мы знаем, что преобразования Лапласа микрофункции, определенной посредством функции $F(s, x)$ в некоторой точке $x$ (скажем, $x=x_{0}$ ), является однозначной функцией.

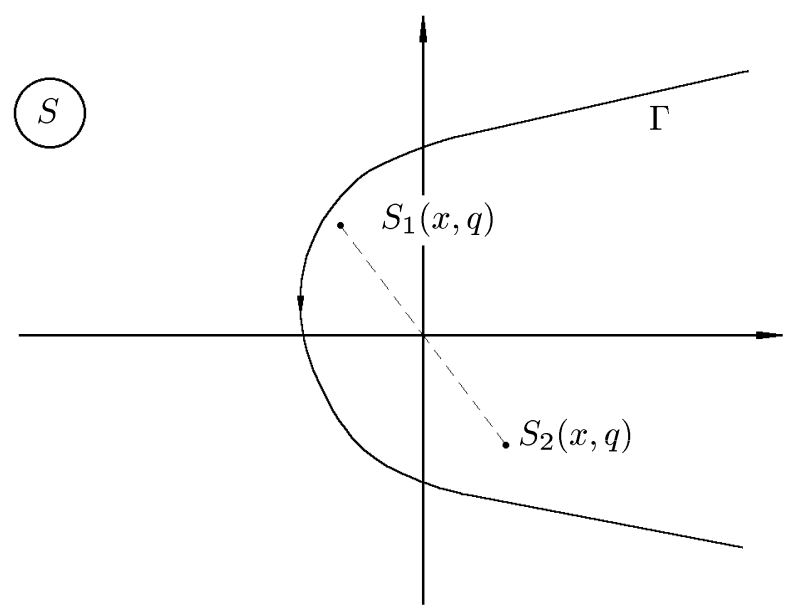

Рис. 4

Для определенности рассмотрим систему ресургентных уравнений для микрофункции, соответствующей рецессивной компоненте функции $u(x, k)$ в точке $x_{0}$. Для того чтобы сделать это, мы положим в системе (18) $F_{0}^{2}=0$. Тогда мы имеем $F_{1}^{2}=\mathscr{A} F_{0}^{2}=0$ и в силу первого из уравнений $(18)$, получаем $F_{0}^{1}=\mathscr{A}^{2} F_{1}^{2}$. Теперь, исключая микрофункцию $F_{1}^{2}$, мы приходим к следующей системе ресургентных уравнений:

$$
\left\{\begin{array}{l}
\Delta\left(\mathscr{A}_{0}^{1}\right)=-\mathscr{A}^{-2} F_{0}^{1}, \\
\Delta\left(\mathscr{A}^{-1} F_{0}^{1}\right)=\mathscr{A}^{2} F_{0}^{1} .
\end{array}\right.
$$


Обозначая через $F_{d}$ и $F_{r}$ соответственно доминантную и рецессивную компоненты в точке $q=q_{1} F_{d}=\mathscr{A} F_{0}^{1}=F_{1}^{1}, F_{r}=\mathscr{A}^{-2} F_{0}^{1}=F_{1}^{2}$, мы можем переписать последнюю систему в следующем виде

$$
\left\{\begin{array}{l}
\Delta F_{d}=-F_{r}, \\
\left(\mathscr{A}^{-1} \Delta \mathscr{A}\right) F_{r}=F_{d} .
\end{array}\right.
$$

Заметим теперь, что оператор $\mathscr{A}^{-1} \Delta \mathscr{A}$ во втором уравнении является ни чем иным, как сторонней производной микрофункции $F_{r}$ в точке $s_{2}(x, q)$ (равно как и оператор $\Delta$ в первом уравнении может считаться сторонней производной микрофункции $F_{d}$ в точке $\left.s_{1}(x, q)\right)$. Таким образом, последняя система может быть рассмотрена как система ресургентных уравнений

$$
\left\{\begin{array}{l}
\Delta_{s_{1}} F_{d}=-F_{r}, \\
\Delta_{s_{2}} F_{r}=F_{d}
\end{array}\right.
$$

при одном и том же значении параметра $x$.

Для того чтобы дать общее решение выведенной выше системы ресургентных уравнений, нам необходимы некоторые факты из теории ресургентных уравнений. Напомним их вкратце (детали см. в [2], [13], [4]). Рассмотрим следующую систему линейных сторонних дифференциальных уравнений с ресургентными коэффициентами

$$
\left\{\begin{array}{l}
\Delta_{s_{1}} F=A_{1}(x) F+B_{1}(x) G, \\
\Delta_{s_{2}} G=A_{2}(x) F+B_{2}(x) G, \\
\Delta_{s} F=0 \text { для } s \neq s_{1} \text { и } \Delta_{s} G=0 \text { для } s \neq s_{2} .
\end{array}\right.
$$

Имеет место следующее утверждение. (Все произведения, используемые ниже, суть произведения в сверточной алгебре $\mathscr{M}_{0, \text { cont }}$ бесконечно продолжимых микрофункций, см. [4].)

Теорема 3. Пусть $\left(F_{1}, G_{1}\right)$ и $\left(F_{2}, G_{2}\right)$ - два решения системы уравнений (20) такие, что определитель

$$
D \stackrel{\text { def }}{=}\left|\begin{array}{ll}
F_{1} & F_{2} \\
G_{1} & G_{2}
\end{array}\right|=F_{1} G_{2}-F_{2} G_{1}
$$

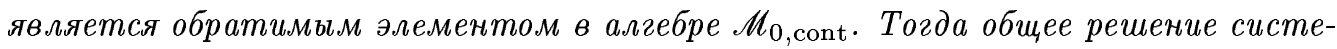
мы (20) записывается в виде

$$
\left(\begin{array}{l}
F \\
G
\end{array}\right)=C_{1}\left(\begin{array}{l}
F_{1} \\
G_{1}
\end{array}\right)+C_{2}\left(\begin{array}{l}
F_{2} \\
G_{2}
\end{array}\right),
$$

где $C_{1}$ и $C_{2}$ - константы ресургениии, т.е. әлементы $C_{1}, C_{2} \in \mathscr{M}_{0, \text { cont }}$ так, что $\Delta_{s} C_{j}=0$ для любого значения $s \in \mathbb{C}$.

Следующий этап в исследовании полученной системы ресургентных уравнений состоит в том, чтобы показать, что классическая функция Эйри (14) является ресургентной функцией, удовлетворяющей системе (19). Производя замену переменных

$$
\left(\xi x-\frac{\xi^{3}}{3}\right)=-s,
$$


мы приведем выражение для функции Эйри $A i(x, k)$ к виду

$$
A i(x, k)=\int_{\gamma^{\prime}} e^{-k s} \frac{d \xi(s, x)}{d s} d s,
$$

где $\xi=\xi(s, x)$ является, вообше говоря, ветвящимся решением уравнения (21) относительно переменной $\xi$. Легко видеть, что особые точки функции $\xi(s, x)$ определяются выражением $s=\frac{2}{3} x^{3 / 2}$, которое совпадает с формулой (13). Далее прямо из определения функции $u(x, k)$ следует, что эта функция является однозначной в окрестности соответствующих фокальных точек (на самом деле, функция, определенная формулой (14), является целой функцией переменной $x)$. Поэтому две микрофункции $F_{d}^{(1)}$ и $F_{r}^{(1)}$, определенные функцией $d \xi(s, x) / d s$, образуют решение ресургентной системы (19).

Поскольку сторонние производные коммутируют с обычными, можно легко построить другое решение $\left(F_{d}^{(2)}, F_{r}^{(2)}\right)$ системы $(19)$, где $F_{d}^{(2)}$ и $F_{r}^{(2)}$ - соответственно доминантньй и рецессивньй компоненты функции $\partial u(x, k) / \partial x$. Соответствуюший детерминант

$$
D=\left|\begin{array}{cc}
F_{d}^{(1)} & F_{d}^{(2)} \\
F_{r}^{(1)} & F_{r}^{(2)}
\end{array}\right|
$$

является преобразованием Бореля вронскиана

$$
J=\left|\begin{array}{ll}
u_{d}(x, k) & \frac{\partial u_{d}(x, k)}{\partial x} \\
u_{r}(x, k) & \frac{\partial u_{r}(x, k)}{\partial x}
\end{array}\right|
$$

где $u_{d}(x, q)$ и $u_{r}(x, q)$ - преобразования Лапласа микрофункций $F_{d}^{(1)}$ и $F_{r}^{(1)}$. Поскольку функции $u_{d}$ и $u_{r}$ - два линейно независимых решения уравнения (15), то функция $J$ является неисчезающей константой, и, следовательно, микрофункция $D$, определенная

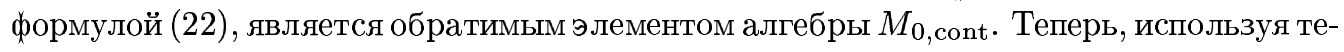
орему 3 , можно записать общее решение системы (19) следующим образом:

$$
\left(\begin{array}{c}
F_{d} \\
F_{r}
\end{array}\right)=C_{1}\left(\begin{array}{c}
F_{d}^{(1)} \\
F_{r}^{(1)}
\end{array}\right)+C_{2}\left(\begin{array}{c}
F_{d}^{(2)} \\
F_{r}^{(2)}
\end{array}\right) .
$$

Здесь $C_{1}$ и $C_{2}-$ произвольные константы ресургенции.

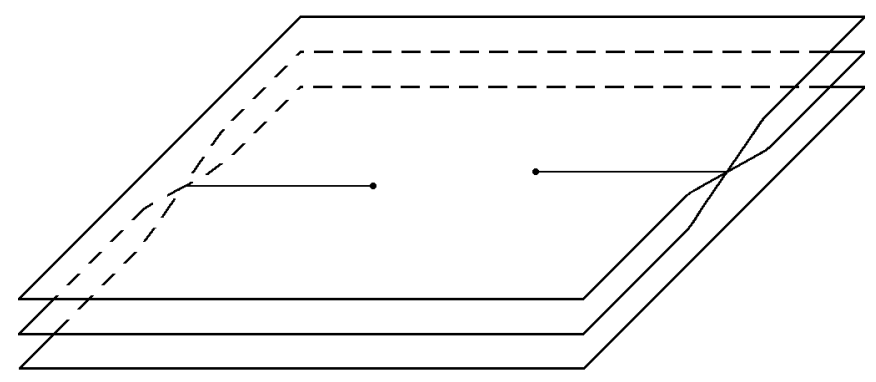

Рис. 5 
Последняя формула позволяет исследовать явление Стокса для решения $\left(F_{d}, F_{r}\right)$ ресургентной системы (19). Действительно, легко видеть, что римановы поверхности всех функций $F_{d}^{(j)}, F_{r}^{(j)}, j=1,2$, такие, какие показаны на рис. 5 . Используя конкретньй вид римановой поверхности, нетрудно вычислить сторонние производные (и, следовательно, связывающие гомоморфизмы) от этих функций. Последняя формула позволяет вычислить сторонние производные и связывающие гомоморфизмы для произвольного решения системы уравнений (19) и, тем самым, исследовать явление Стокса для этого решения.

4.2. Функции параболического цилиндра. В этом пункте мы изучим интегралы Лапласа с ресургентной структурой, совпадающей с ресургентной структурой функций параболического цилиндра (такие функции назьваются также функииями Вебера). Мы напомним, что эти функции определяются как решения уравнения Вебера

$$
k^{-2} \frac{d^{2} u}{d x^{2}}-x^{2} u=0
$$

и имеют следующее интегральное представление:

$$
u(x, k)=k^{1 / 2} \int_{\gamma} e^{k\left(2 p^{2} x+x^{2} / 2+p^{4}\right)} d p .
$$

Контур $\gamma$ в последнем выражении выбирается таким образом, чтобы экспонента, стоящая под знаком интеграла, убьвала вдоль контура, когда $|p| \rightarrow \infty$. Как и выше, замена переменных

$$
s=-\left(2 p^{2} x+\frac{x^{2}}{2}+p^{4}\right)
$$

приводит к следующему выражению:

$$
u(x, q)=k^{1 / 2} \int_{\gamma} e^{-k s} \frac{d p}{d s}(s, x) d s .
$$

Функция $p=p(s, x)$ вычисляется явным образом

$$
p(s, x)=\sqrt{-x+\sqrt{s+\frac{x^{2}}{2}}}
$$

и, как легко видеть, имеет две точки ветвления $s= \pm x^{2} / 2$. Риманова поверхность этой функции показана на рис. 6. Можно легко проверить, что две микрофункции, соответствующие особой точке $s=-x^{2} / 2$, отличаются друг от друга только зна́ком и, следовательно, формула (24) доставляет два линейно независимых решения уравнения (23), если мы выберем два контура $\gamma$ таким образом, чтобы выражение (24) являлось преобразованием Лапласа микрофункций, определенных посредством функции $p(s, x)$ в точ$\operatorname{kax} s= \pm x^{2} / 2$.

Таким образом, мы построили два линейно независимых ресургентных решения уравнения Вебера с ресургентной структурой

$$
s= \pm \frac{x^{2}}{2}
$$




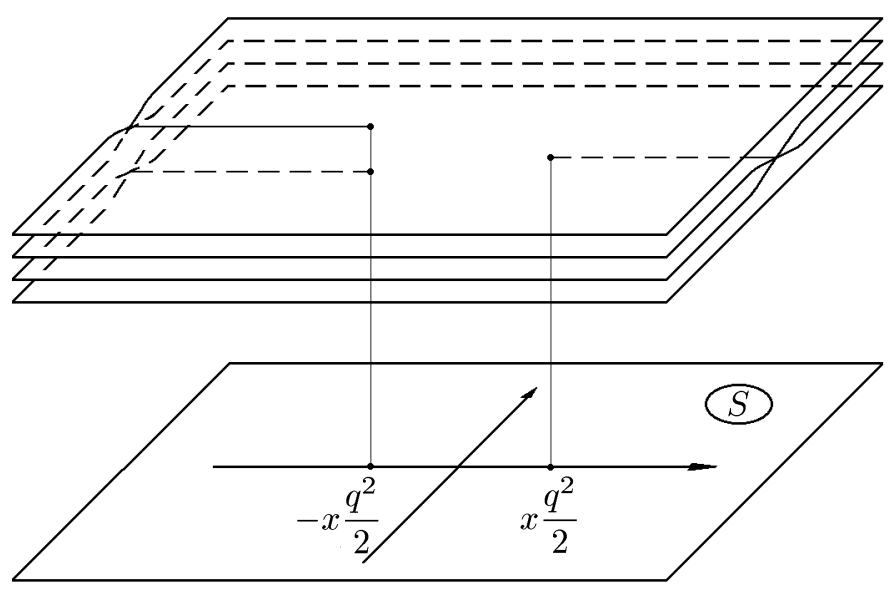

Рис. 6

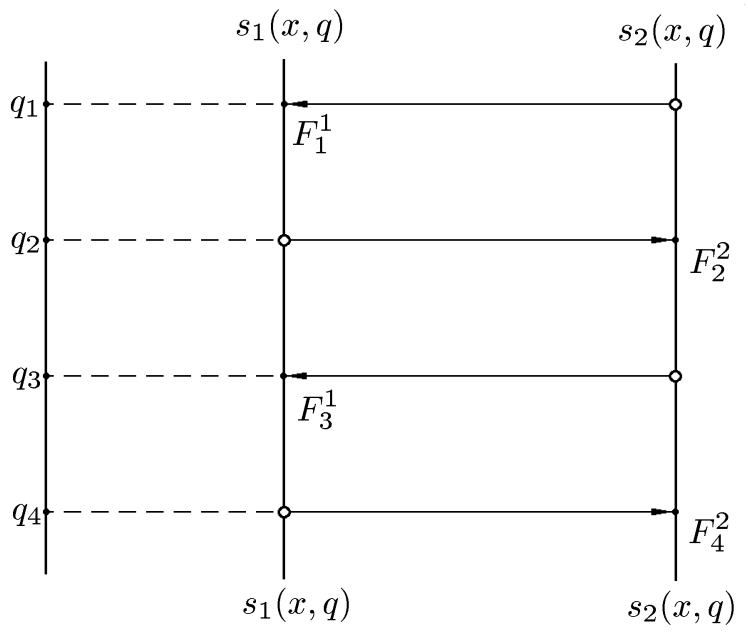

Рис. 7

Исследуем теперь интегралы Лапласа общего вида

$$
u(x, k)=\int_{\gamma} e^{k S(x, \xi)} a(x, \xi) d \xi
$$

с данной ресургентной структурой (25). Диаграмма освешения показана на рис. 7. Соответствующая система ресургентных уравнений имеет вид

$$
\left\{\begin{array}{l}
F_{1}^{1}=\mathscr{A}^{2} F_{3}^{1}-\Delta\left(\mathscr{A} F_{4}^{2}\right), \\
F_{3}^{1}=\mathscr{A}^{2} F_{1}^{1}-\Delta\left(\mathscr{A} F_{2}^{2}\right), \\
F_{2}^{2}=\mathscr{A}^{2} F_{4}^{2}-\Delta\left(\mathscr{A} F_{1}^{1}\right), \\
F_{4}^{2}=\mathscr{A}^{2} F_{2}^{2}-\Delta\left(\mathscr{A} F_{3}^{1}\right) .
\end{array}\right.
$$


Для того чтобы записать систему ресургентных уравнений для доминантной (в точке $x_{1}$ ) микрофункции, мы положим $F_{1}^{1}=0$ и получим следующую систему:

$$
\left\{\begin{array}{l}
\mathscr{A}^{-1} \Delta\left(\mathscr{A} F_{3}^{1}\right)=\mathscr{A}^{3} F_{4}^{2}-\mathscr{A}^{-1} F_{4}^{2} \\
\mathscr{A}^{-2} \Delta\left(\mathscr{A} F_{4}^{2}\right)=-F_{3}^{1} \\
\Delta\left(\mathscr{A}^{3} F_{4}^{2}\right)=F_{3}^{1} .
\end{array}\right.
$$

Обозначая $G_{1}=F_{3}^{1}, G_{2}^{\prime}=\mathscr{A}^{3} F_{4}^{2}$ и $G_{2}^{\prime \prime}=\mathscr{A}^{-1} F_{4}^{2}$, мы приведем последнюю систему к виду

$$
\left\{\begin{aligned}
\Delta_{s_{1}} G_{1} & =G_{2}^{\prime}-G_{2}^{\prime \prime} \\
\Delta_{s_{2}} G_{2}^{\prime} & =G_{1} \\
\Delta_{s_{2}} G_{2}^{\prime \prime} & =-G_{1}
\end{aligned}\right.
$$

Видно, что полученная система отличается от системы, построенной в предыдущем примере, тем, что две микрофункции $G_{2}^{\prime}$ и $G_{2}^{\prime \prime}$ имеют особенность в одной и той же точке. Причину этого феномена можно усмотреть из рис. 6 , где показана риманова поверхность типичного решения построенной системы ресургентных уравнений. В самом деле, очевидно, что функция с такой римановой поверхностью определяет в одной из своих особых точек две (вообще говоря, различные) микрофункции.

Построим теперь общее решение системы (26). Прежде всего, добавляя второе уравнение этой системы к третьему, мы получим соотношение $\Delta_{s_{2}}\left(G_{2}^{\prime}+G_{2}^{\prime \prime}\right)=0$. Отсюда следует, что функция $G_{2}^{\prime}+G_{2}^{\prime \prime}-$ константа ресургенции:

$$
G_{2}^{\prime}+G_{2}^{\prime \prime}=C_{1}
$$

Исключая микрофункцию $G_{2}^{\prime \prime}$ из системы $(26)$ с помощью последнего соотношения, получим

$$
\left\{\begin{array}{l}
\Delta_{s_{1}} G_{1}=2 G_{2}^{\prime}-C_{1} \\
\Delta_{s_{2}} G_{2}^{\prime}=G_{1}
\end{array}\right.
$$

Далее подстановка $G_{2}^{\prime}=G_{2}^{\prime \prime \prime}+C_{1} / 2$ приводит рассматриваемую систему к виду

$$
\left\{\begin{array}{l}
\Delta_{s_{1}} G_{1}=2 G_{2}^{\prime \prime \prime} \\
\Delta_{s_{2}} G_{2}^{\prime \prime \prime}=G_{1}
\end{array}\right.
$$

Нетрудно проверить, что две микрофункции $H_{1}^{(1)}$ и $H_{2}^{(1)}$, определенные с помощью функций $(24)$, равно как и две микрофункции $H_{1}^{(2)}$ и $H_{2}^{(2)}$, определенные с помощью производных функции (24), удовлетворяют последней системе. Рассуждения, аналогичные предыдущему пункту, показывают, что общее решение системы уравнений (27) может быть записано следующим образом:

$$
\left(\begin{array}{c}
G_{1} \\
G_{2}^{\prime \prime \prime}
\end{array}\right)=C_{2}\left(\begin{array}{c}
H_{1}^{(1)} \\
H_{2}^{(1)}
\end{array}\right)+C_{3}\left(\begin{array}{c}
H_{1}^{(2)} \\
H_{2}^{(2)}
\end{array}\right) .
$$


Таким образом, общее решение системы (26) имеет вид

$$
\left\{\begin{array}{l}
G_{1}=C_{2} H_{1}^{(1)}+C_{3} H_{1}^{(2)} \\
G_{2}^{\prime}=\frac{C_{1}}{2}+C_{2} H_{2}^{(1)}+C_{3} H_{2}^{(2)} \\
G_{2}^{\prime \prime}=\frac{C_{1}}{2}-C_{2} H_{2}^{(1)}-C_{3} H_{2}^{(2)}
\end{array}\right.
$$

где $C_{j}, j=1,2,3,-$ произвольные константы ресургенции. Очевидно, что для описания общего вида функции с ресургентной структурой веберовского типа нужно к полученному ранее решению добавить аналогичное решение, отвечающее случаю, когда точки $s=s_{1}(x, q)$ и $s=s_{2}(x, q)$ переставлены.

Мы оставляем соответствующие вычисления читателю. Пожалуй, единственное, что следует упомянуть в заключение этого пункта, это то, что в отличие от предыдущего примера, полученная здесь ресургентная система имеет в качестве решения константу ресургенции. Причиной этого является то, что монодромия особенных точек, соответствующих ресургентньм функциям веберовского типа, тривиальна, и поэтому мы получаем соответствующее тривиальное решение ресургентной системы.

Московский государственный университет им. М.В.Ломоносова

Поступило

Институт прикладной математики $\mathrm{PAH}$

\section{СПИСОК ЦИТИРОВАННОЙ ЛИТЕРАТУРЫ}

[1] Écalle J. Les Fonctions Résurgentes. I, II, III. Paris: Publ. Mathématiques d'Orsay, 1981-1985.

[2] Candelpergher B., Nosmas J. C., Pham F. Approche de la Résurgence. Paris: Hermann, 1993.

[3] Sternin B., Shatalov V. On a notion of resurgent function of several variables // Math. Nachr. 1995. V. 171. P. 283-301.

[4] Sternin B., Shatalov V. Borel-Laplace Transform and Asymptotic Theory. Florida: CRC Press, 1995.

[5] Berry M. V., Howls C. J. Hyperasymptotics for integrals with saddles // Proc. Roy. Soc. London. Ser. A. 1991. V. 443. P. 657-675.

[6] Malgrange B. Méthode de la phase stationnaire et sommation de Borel // Complex Analysis. Microlocal Calculus and Relativistic Quantum Theory. Lecture Notes in Phys. V. 126. Berlin-Heidelberg: Springer Verlag, 1980.

[7] Pham F. Vanishing homologies and the $n$ variable saddlepoint method // Proc. Sympos. Pure Math. V. 40. Providence: AMS, 1983. P. 319-333. Part 2.

[8] Sternin B., Shatalov V. Saddle Point Method and Resurgent Analysis. Preprint № 95-69. Bohn: Max-Planck-Institut für Mathematik, 1995.

[9] Sternin B., Shatalov V. Differential Equations on Complex Manifolds. Dordrecht: Kluwer Acad. Publ., 1994.

[10] Федорюк М. В. Метод перевала. М.: Наука, 1977.

[11] Tougeron J.-C. An introduction to the theory of Gevrey expansions and to Borel-Laplace transform with some applications. Preprint. Canada: Univ. of Toronto, 1990.

[12] Lefschetz S. L'analysis Situs et la Géométrie Algébrique. Paris: Gauthier-Villars, 1924.

[13] Delabaere E. Introduction to the Écalle theory // Computer Algebra and Differential Equations / ed. E. Tournier. London Math. Soc. Lecture Note Ser. V. 193. Cambridge: Univ. Press, 1994. P. 59-102. 